\title{
Competition between electromagnetically induced transparency and Raman processes
}

\author{
G. S. Agarwal* and T. N. Dey ${ }^{\dagger}$ \\ Department of Physics, Oklahoma State University, Stillwater, Oklahoma 74078, USA \\ Daniel J. Gauthier \\ Department of Physics, Duke University, Durham, North Carolina 27708, USA
}

(Received 22 May 2006; published 5 October 2006)

\begin{abstract}
We present a theoretical formulation of the competition between electromagnetically induced transparency (EIT) and Raman processes. The latter become important when the medium can no longer be considered to be dilute. Unlike the standard formulation of EIT, we consider all fields applied and generated as interacting with both the transitions of the $\Lambda$ scheme. We solve the Maxwell equations for the net generated field using a fast-Fourier-transform technique and obtain predictions for the probe, control, and Raman fields. We show how the intensity of the probe field is depleted at higher atomic number densities due to the buildup of multiple Raman fields. Furthermore, we find that the generated fields and the input fields acquire oscillatory behavior as a function of the density of the medium due to dynamical coupling of the various Raman processes.
\end{abstract}

DOI: $10.1103 /$ PhysRevA.74.043805

PACS number(s): 42.50.Gy, 42.65.-k

A multilevel atomic system interacting with several electromagnetic fields can give rise to a variety of phenomena that depend on the strength and detunings of the fields. Often, the various processes compete with each other, whereby some processes are suppressed or interference between processes renders the medium transparent to the applied fields [1]. Well-known examples include competition between third-harmonic generation and multiphoton ionization [2], and between four-wave mixing and two-photon absorption $[3,4]$. Very recently, Harada et al. demonstrated experimentally that stimulated Raman scattering can disrupt electromagnetically induced transparency (EIT), where the incident probe beam is depleted and new fields are generated via Raman processes [5]. The disruption of EIT is important to understand because it may degrade the performance of EITbased applications, such as optical memories and buffers, and magnetometers. In this paper we present a theoretical formulation that enables us to study the competing EIT and various orders of Raman processes to all orders in the applied and generated fields.

The standard treatment of EIT [6] is based on the scheme of Fig. 1, where the atoms in the state $|c\rangle$ interact with a probe field of frequency $\omega$. A control field of frequency $\omega_{c}$ interacts on the unoccupied transition $|a\rangle \leftrightarrow|b\rangle$. The probe and the control fields are tuned such that

$$
\omega-\omega_{c}=\omega_{b c} .
$$

This results in no absorption of the probe field provided the coherence $\rho_{b c}$ has no decay. This treatment assumes that the frequency separation $\omega_{b c}$ is so large that the interaction of the control field $\omega_{c}$ (probe field $\omega$ ) with the transition $|a\rangle \leftrightarrow|b\rangle$ $(|a\rangle \leftrightarrow|c\rangle)$ can be ignored. At high atomic number densities

\footnotetext{
*On leave of absence from Physical Research Laboratory, Navrangpura, Ahmedabad 380 009, India.

†Electronic address: tarak.dey@okstate.edu
}

or for strong fields, this approximation no longer holds, which is the situation we consider here.

At higher densities, Raman processes start becoming important $[7,8]$, such as those shown in Fig 2, for example. The Raman generation of the fields at $\omega_{c}-\omega_{b c}, \omega+\omega_{b c}$ can further lead to newer frequencies like $\omega_{c}-2 \omega_{b c}$. In order to account for the Raman processes, we write the electromagnetic field acting on both the transitions as

$$
E(t)=\mathcal{E}(t) e^{-i \omega_{c} t},
$$

where $\mathcal{E}(t)$ denotes the net generated field. At the input face of the medium $\mathcal{E}(t)$ has two components to account for both control and probe fields,

$$
\mathcal{E}(t)=\mathcal{E}_{c}+\mathcal{E}_{p} e^{-i\left(\omega-\omega_{c}\right) t}
$$

Under the Raman-resonance condition (1), we expect $\mathcal{E}(t)$ to have the structure

$$
\mathcal{E}(t)=\sum \mathcal{E}^{(n)} e^{-i n \omega_{b c} t}
$$

Thus, $\mathcal{E}^{(-1)}$ gives the strength of the Stokes process of Fig. $2(\mathrm{a}) ; \mathcal{E}^{(+2)}$ gives the strength of the process of Fig. 2(b); and $\mathcal{E}^{(+1)}$ describes the changes in the probe field. For low atomic number densities, we expect the usual results and therefore $\mathcal{E}^{(+1)} \approx \mathcal{E}$ and $\mathcal{E}^{(0)} \approx \mathcal{E}_{c}$.

To calculate the net generated field $\mathcal{E}(t)$ for arbitrary atomic number density, we have to solve the coupled Maxwell and density matrix equations. We consider now the situation as shown schematically in Fig. 3. The applied field $E(z, t)$ couples the excited state $|a\rangle$ to both ground states $|b\rangle$ and $|c\rangle$. Here the $2 \gamma$ 's represent rates of spontaneous emission. In a frame rotating with the frequency $\omega_{c}$ the density matrix equations for the atomic system are given by

$$
\begin{gathered}
\dot{\rho}_{a a}=i \Omega_{\mathcal{E}}\left(\rho_{b a}+\rho_{c a}\right)-i \Omega_{\mathcal{E}}^{*}\left(\rho_{a b}+\rho_{a c}\right)-4 \gamma \rho_{a a}, \\
\dot{\rho}_{b b}=i \Omega_{\mathcal{E}}^{*} \rho_{a b}-i \Omega_{\mathcal{E}} \rho_{b a}+2 \gamma \rho_{a a}, \\
\dot{\rho}_{a b}=-\left(2 \gamma-i \Delta_{c}\right) \rho_{a b}+i \Omega_{\mathcal{E}}\left(\rho_{b b}-\rho_{a a}\right)+i \Omega_{\mathcal{E}} \rho_{c b},
\end{gathered}
$$




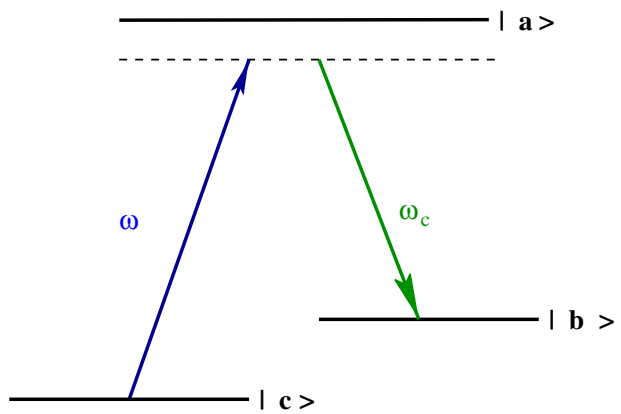

FIG. 1. (Color online) A schematic diagram of a three-level atomic system with energy spacing $\hbar \omega_{b c}$ between two ground states $|c\rangle$ and $|b\rangle$. The control field with frequency $\omega_{c}$ and probe field with frequency $\omega$ act on the atomic transitions $|a\rangle \leftrightarrow|b\rangle$ and $|a\rangle \leftrightarrow|c\rangle$, respectively.

$$
\begin{gathered}
\dot{\rho}_{a c}=-\left[2 \gamma-i\left(\Delta_{c}-\omega_{b c}\right)\right] \rho_{a c}+i \Omega_{\mathcal{E}} \rho_{b c}+i \Omega_{\mathcal{E}}\left(\rho_{c c}-\rho_{a a}\right), \\
\dot{\rho}_{b c}=-\left(\Gamma_{b c}+i \omega_{b c}\right) \rho_{b c}+i \Omega_{\mathcal{E}}^{*} \rho_{a c}-i \Omega_{\mathcal{E}} \rho_{b a},
\end{gathered}
$$

where the detuning $\Delta_{c}$ and the space- and time-dependent Rabi frequency $\Omega_{\mathcal{E}}$ of the generated fields are defined by

(a)
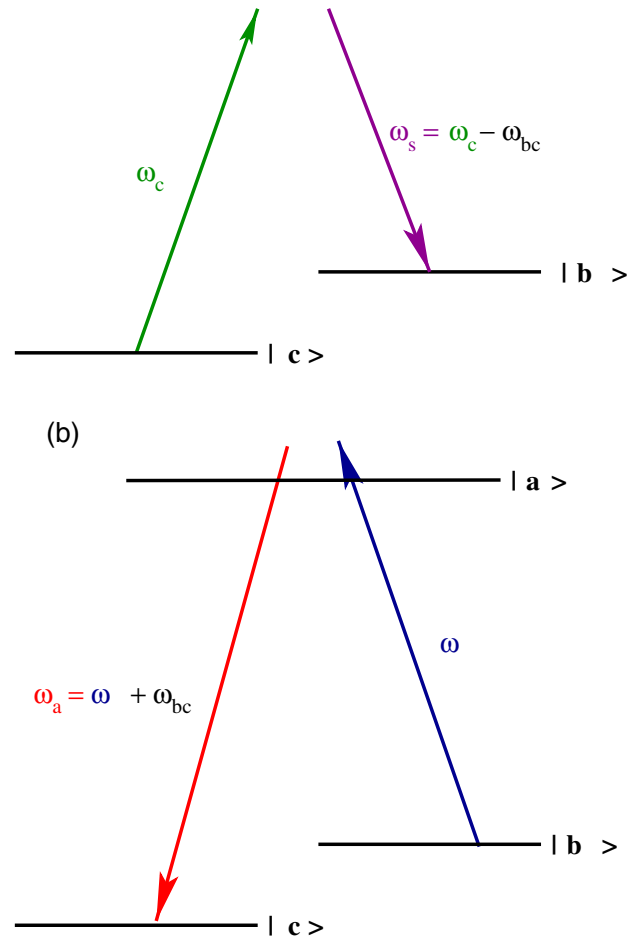

FIG. 2. (Color online) Diagrammatic explanation of the (a) Stokes and (b) anti-Stokes processes. The intermediate state is denoted by $|a\rangle$.

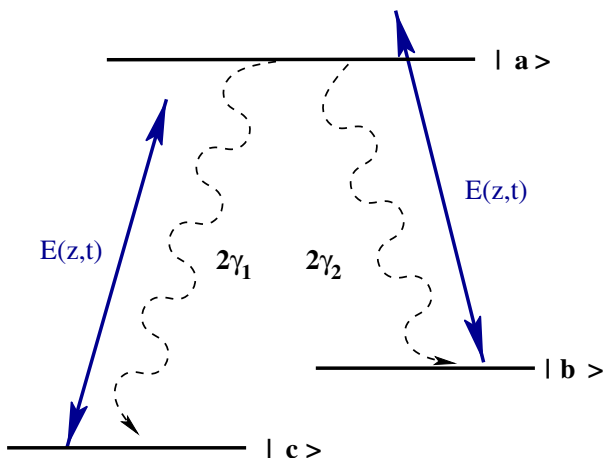

FIG. 3. (Color online) Three-level $\Lambda$ system interacting with the space-time-dependent field $E(z, t)$ on both the optical transitions.

$$
\Delta_{c}=\omega_{c}-\omega_{a c}, \quad \Omega_{\mathcal{E}}(z, t)=\frac{\vec{d} \cdot \overrightarrow{\mathcal{E}}}{\hbar}
$$

In Eqs. (5) the decay of the off-diagonal element $\rho_{b c}$ is given by $\Gamma_{b c}$. Usually it is much smaller than the radiative decay [9]. However, addition of a buffer gas can make this decay prominent. For simplicity, we have assumed $\vec{d}_{a b}=\vec{d}_{a c}=\vec{d}$. The elements $\rho_{a c}$ and $\rho_{a b}$ in the original frame can be obtained by multiplying the solution of Eqs. (5) by $e^{-i \omega_{c} t}$. The induced polarization $\overrightarrow{\mathcal{P}}$ is given by

$$
\overrightarrow{\mathcal{P}}=\left(\vec{d} \rho_{a b}+\vec{d} \rho_{a c}\right) e^{-i \omega_{c} t}
$$

The Maxwell equations in the slowly varying envelope approximation lead to the following equation for the generated field:

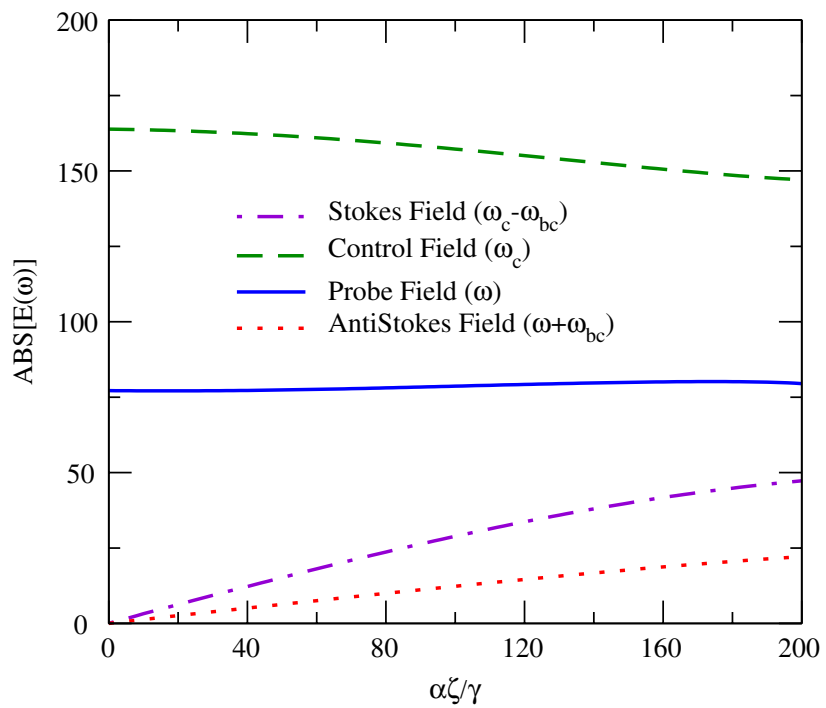

FIG. 4. (Color online) Amplitudes of different Fourier components of the net generated field are plotted as a function of the atomic density of the medium. The normalized propagation length $\alpha \zeta / \gamma=200$ is equivalent to the actual length of the medium $L$ $=7.13 \mathrm{~cm}$ with an atomic density of $n=2 \times 10^{10}$ atoms $/ \mathrm{cm}^{3}$. The other parameters of the above graph are chosen as follows: input control laser Rabi frequency $\Omega_{\mathcal{E}}=0.5 \gamma, \mathcal{E}_{p} / \mathcal{E}_{c}=0.5, \Delta_{c}=0.0, \gamma$ $=9.475 \times 10^{6}, \Gamma_{b c}=0.0, \omega_{b c}=100 \gamma$, and $\lambda=766.4 \mathrm{~nm}$. 


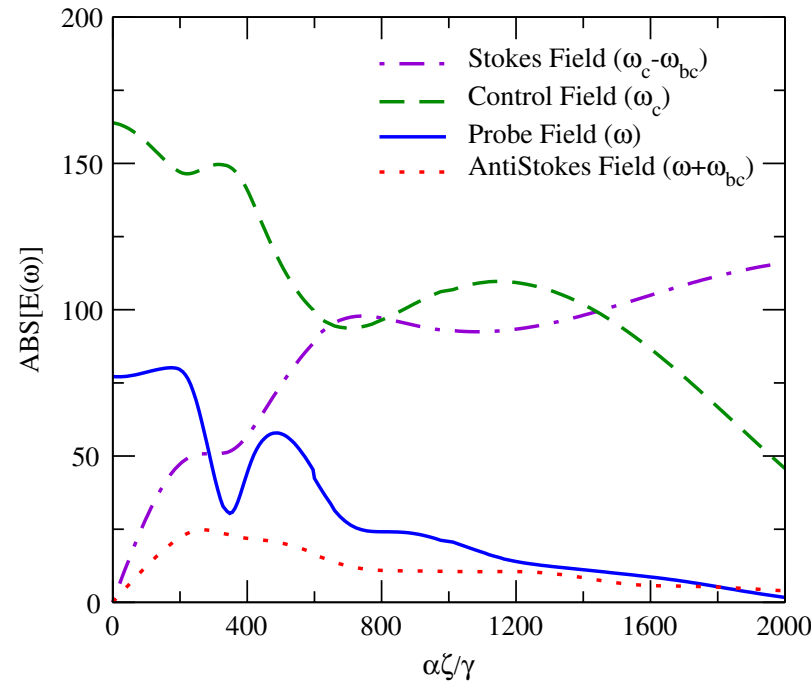

FIG. 5. (Color online) The spectral amplitudes of different fields are plotted as a function of the atomic density of the medium. The normalized propagation length $\alpha \zeta / \gamma=2000$ is equivalent to the actual length of the medium $L=7.1 \mathrm{~cm}$ when the atomic density $n=2 \times 10^{11}$ atom $/ \mathrm{cm}^{3}$. The other parameters are chosen as follows: input control laser Rabi frequency $\Omega_{\mathcal{E}}=0.5 \gamma, \mathcal{E}_{p} / \mathcal{E}_{c}=0.5, \Delta_{c}=0.0$, $\Gamma_{b c}=0.0$, and $\omega_{b c}=100 \gamma$.

$$
\left(\frac{\partial \Omega_{\mathcal{E}}}{\partial z}+\frac{\partial \Omega_{\mathcal{E}}}{\partial c t}\right)=i \frac{\alpha}{2}\left(\rho_{a c}+\rho_{a b}\right)
$$

where $\alpha$ is given by

$$
\alpha=3 \lambda^{2} n \gamma / 4 \pi
$$

and $n$ is the atomic density. The coupled equations (5) and (8) are solved in the moving coordinate system

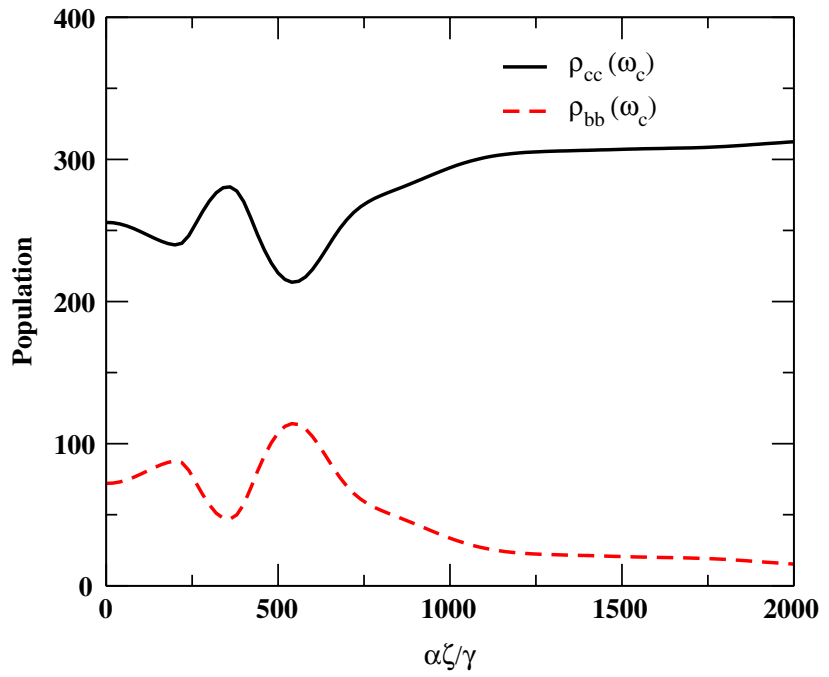

FIG. 6. (Color online) The spectral decompositions of the populations of the levels $|b\rangle$ and $|c\rangle$ at the control laser frequency $\omega_{c}$ are shown as a function of the atomic density of the medium. The other parameters are chosen as follows: input control laser Rabi frequency $\Omega_{\mathcal{E}}=0.5 \gamma, \mathcal{E}_{p} / \mathcal{E}_{c}=0.5, \Delta_{c}=0.0, \Gamma_{b c}=0.0$, and $\omega_{b c}=100 \gamma$.

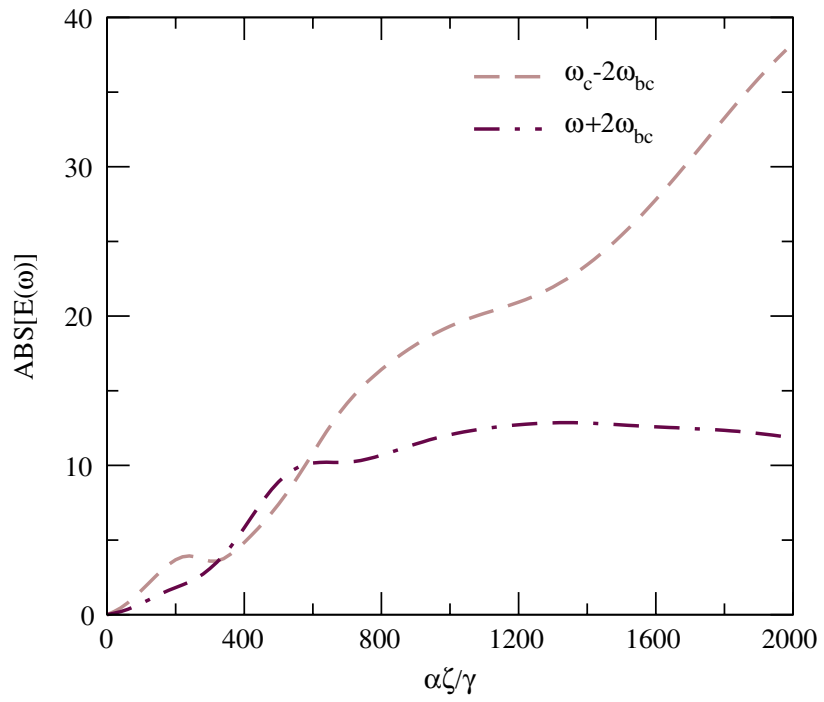

FIG. 7. (Color online) Plot of amplitudes of hyper-Raman components at frequencies $\left(\omega_{c}-2 \omega_{b c}\right),\left(\omega+2 \omega_{b c}\right)$ as a function of the atomic density. All parameters are the same as in Fig. 5.

$$
\tau=t-\frac{z}{c}, \quad \zeta=z
$$

We have numerically solved the coupled set of equations when all the atoms are initially in the state $|c\rangle$ and when the fields at the input face of the medium are given by (3). We calculate $\mathcal{E}(l, \tau)$ and do a fast Fourier transform to obtain the different Fourier components of the field at the output face of the medium. This procedure enables us to find how the probe and control fields evolve and determine when the Raman

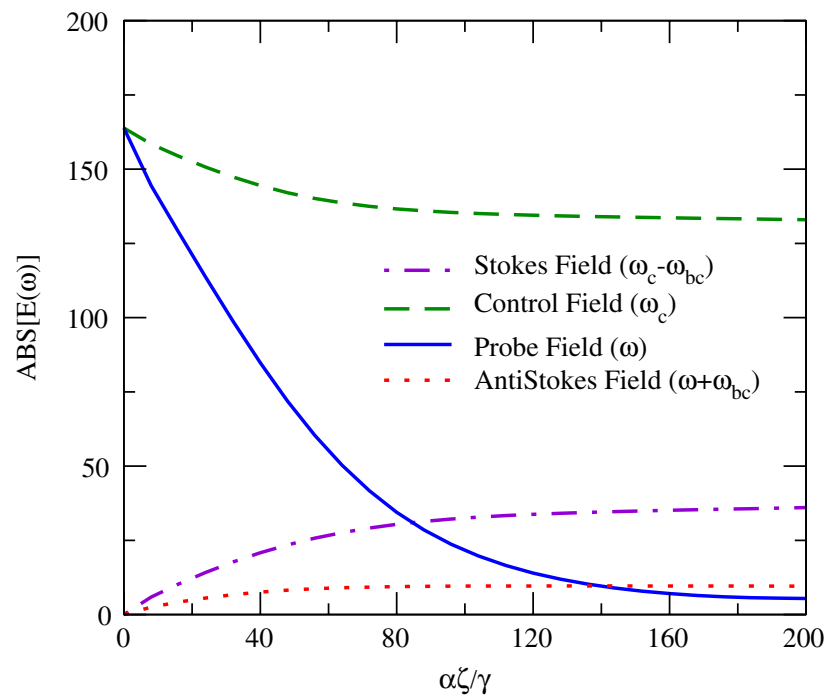

FIG. 8. (Color online) Plot of amplitudes of Stokes, control, probe, and anti-Stokes fields as a function of the atomic number density in the presence of a buffer gas. Here, we have scaled the amplitudes of probe, Stokes, and anti-Stokes fields by a factor of 2 . The other parameters are chosen as follows: density $n=2$ $\times 10^{10}$ atom $/ \mathrm{cm}^{3}$, input control laser Rabi frequency $\Omega_{\mathcal{E}}=0.5 \gamma$, $\mathcal{E}_{p} / \mathcal{E}_{c}=0.5, \Delta_{c}=0.0, \Gamma_{b c}=0.01 \gamma$, and $\omega_{b c}=100 \gamma$. A nonzero value of $\Gamma_{b c}$ accounts for the buffer gas. 
processes become important. In the simulations, we have used parameters [8] that are different from those in the experiment [5] to avoid numerical problems [10]. What we demonstrate here is that the overall behavior of the fields is in broad agreement with the observations despite the differences in parameters. Furthermore, we make predictions about both probe and control fields as well as about the generation of higher-order Raman processes.

We now present the results of numerical calculations for EIT vs Raman processes. In Fig. 4, we show results for the low-density regime. In this region, we notice almost no change in the probe field and thus EIT dominates. It is also seen that the Raman processes slowly start to build up, leading to a drop in the control field amplitude.

We next consider the high-density regime, as shown in Fig. 5. This is the region when multiple Raman processes build up significantly [11]. Our numerical results are in broad agreement with the observations of Harada et al. [5], where they observe the depletion of the probe field and the generation of the Stokes field at $\left(\omega_{c}-\omega_{b c}\right)$. In particular, we see in Fig. 5 that the generation of radiation at $\left(\omega_{c}-\omega_{b c}\right)$ is very important and the probe beam is depleted. We also notice an additional feature - the probe exhibits some oscillatory character before dying out. This oscillation is due to the fact that any population that is transferred to the state $|b\rangle$ can produce a field at the probe frequency via the Raman process. When this happens, the control field amplitude falls. In general, all the components are coupled to each other and evolve dynamically as shown in Fig. 5. There is a correlation between the behavior of the fields and the atomic populations. In Fig. 6 , we show, for instance, the Fourier components of the populations of the levels $b$ and $c$ at the control laser frequency. The conversion of the control laser into the Stokes field is well reflected by the changes in the population $\rho_{c c}\left(\omega_{c}\right)$ until the reverse process starts taking place. Furthermore, when enough population is back in level $b$, the Stokes wave starts to build back up again. The oscillatory character is a reflection of the fact that all processes are essentially occurring at the same time. In Fig. 7, we show the buildup of several hyper-Raman processes. Note that the linearized theory of Harada et al. [5] does not contain information on such a buildup.

Finally we discuss the effect of a buffer gas on the generated field. This is done by choosing $\Gamma_{b c}$ in Eq. (5) to be nonzero [12]. The rate $\Gamma_{b c}$ is proportional to the pressure of the buffer gas. The results are shown in Fig. 8, where it is seen that the amplitude of the probe field depletes faster in the presence of a buffer gas. This is because the EIT dip in the atomic response does not go to zero and there is net absorption of the probe [6]. On comparison of Figs. 5 and 8, we see that the amplitudes of the probe field and the generated Raman field become equal at $\alpha \zeta / \gamma=272$ (without buffer gas) and 84 (with buffer gas). This is in agreement with the observation in Ref. [5].

In conclusion, we have investigated the competition between electromagnetically induced transparency and Raman processes in a $\Lambda$ system due to the cross talk among the optical transitions. We have demonstrated that the EITinduced probe spectrum is very pronounced in comparison to the higher-order Raman sidebands for a low atomic number density. However, the generated Raman fields become dominant for an atomic number density that is only ten times higher.
[1] D. J. Gauthier, J. Chem. Phys. 99, 1618 (1993).

[2] S. P. Tewari and G. S. Agarwal, Phys. Rev. Lett. 56, 1811 (1986), and references therein.

[3] M. S. Malcuit, D. J. Gauthier, and R. W. Boyd, Phys. Rev. Lett. 55, 1086 (1985); R. W. Boyd, M. S. Malcuit, D. J. Gauthier, and K. Rzażewski Phys. Rev. A 35, 1648 (1987).

[4] G. S. Agarwal, Phys. Rev. Lett. 57, 827 (1986).

[5] K. I. Harada, T. Kanbashi, M. Mitsunaga and K. Motomura, Phys. Rev. A 73, 013807 (2006). These authors examine their experiments using an analysis based on linearized equations for probe and Stokes fields and no depletion of the control field. In our work we treat all fields on equal basis as the depletion of the control field could be important, and several higher-order Raman processes become active.

[6] S. E. Harris, J. E. Field, and A. Imamoglu, Phys. Rev. Lett. 64, 1107 (1990); S. E. Harris, Phys. Today 50(7),36 (1997).

[7] M. Poelker and P. Kumar, Opt. Lett. 17, 399 (1992); M. Poelker, P. Kumar, and S.-T. Ho, ibid. 16, 1853 (1991); M. T. Gruneisen, K. R. MacDonald, and R. W. Boyd, J. Opt. Soc. Am. B 5, 123 (1988); P. Kumar and J. H. Shapiro, Opt. Lett. 10, 226 (1985).

[8] H. M. Concannon, W. J. Brown, J. R. Gardner, and D. J. Gauthier, Phys. Rev. A 56, 1519 (1997).
[9] I. Novikova, A. B. Matsko, V. L. Velichansky, M. O. Scully, and G. R. Welch, Phys. Rev. A 63, 063802 (2001); D. Budker, V. Yashchuk, and M. Zolotorev, Phys. Rev. Lett. 81, 5788 (1998); D. Budker, D. F. Kimball, S. M. Rochester, V. V. Yashchuk, and M. Zolotorev, Phys. Rev. A 62, 043403 (2000).

[10] In the case of sodium the separation of the two hyperfine components is about $2 \mathrm{GHz}$, which is extremely large for our numerical simulations of the coupled Maxwell-Bloch equations. For the same reasons we are currently not able to handle inhomogeneous broadening.

[11] The generation of multiple Raman sidebands has been considered under the condition that the excited state is far detuned from any of the exciting frequencies; see A. V. Sokolov, D. D. Yavuz, and S. E. Harris, Opt. Lett. 24, 557 (1999); K. Hakuta, M. Suzuki, M. Katsuragawa, and J. Z. Li, Phys. Rev. Lett. 79, 209 (1997). Furthermore, Raman generation in a coherently prepared medium has been considered by A. F. Huss, N. Peer, R. Lammeggar, E. A. Korsunsky, and L. Windholz, Phys. Rev. A 63, 013802 (2000).

[12] L. J. Rothberg and N. Bloembergen, Phys. Rev. A 30, 820 (1984); L. J. Rothberg, in Progress in Optics, edited by E. Wolf (Elsevier, Amsterdam, 1987), Vol. 21, pp. 39-101. 